\title{
Preoperative Evaluation of Substernal Goiter by Computed Tomography in the Extended Neck Position
}

\author{
Teruhisa Yano Takuro Okada Hiroki Sato Ryota Tomioka \\ Kiyoaki Tsukahara
}

Department of Otorhinolaryngology, Head and Neck Surgery, Tokyo Medical University, Tokyo, Japan

\section{Keywords}

Substernal goiter · Transcervical approach · Sternotomy · Extended neck computed tomography

\begin{abstract}
Sternotomy is indicated when a goiter cannot be resected via a cervical incision, such as in the case of a substernal goiter extending beyond the aortic arch. In this article, we report a case of a large substernal goiter that was successfully removed using the cervical approach only. This is a case of a 68-year-old woman, diagnosed with goiter 20 years ago, who complained of a neck mass enlargement with associated cough. Pathological examination revealed no malignancy. Computed tomography (CT) scan showed an 11- $\mathrm{cm}$ thyroid mass reaching the level of the aortic arch. Preoperatively, we evaluated the substernal extent of the goiter via CT in the extended neck position to decide whether sternotomy was necessary. With the patient's neck extended, the goiter withdraws cranially above the aortic arch. The mass was then removed via the cervical approach without sternotomy. Preoperative $\mathrm{CT}$ in the extended neck position was thus deemed helpful in deciding whether or not sternotomy was required.
\end{abstract}

\section{Karger $\stackrel{2}{\circ}$}




\section{Introduction}

Goiter is defined as the abnormal enlargement of the thyroid gland, typically in a benign thyroid disease [1]. Surgery is indicated when the goiter is suspicious for malignancy, causes excessive levels of thyroid hormone, measures $>4 \mathrm{~cm}$, or extends into the substernal space [2]. In cases of substernal goiters, sternotomy is usually performed in addition to the cervical approach. While sternotomy has been done in cases of thyroid cancer involving mediastinal lymph nodes, some cases were managed using the cervical approach only [3]. However, the preoperative decision to conduct sternotomy remains a challenge. We report a case wherein preoperative computed tomography (CT) in the extended neck position was helpful in surgical planning. The substernal goiter was presumed to withdraw cranially in the extended neck position during surgery, resulting in successful removal without sternotomy.

\section{Case Report/Case Presentation}

A 68-year-old woman with a chronic persistent cough presented with enlargement of a left neck tumor, diagnosed as goiter 20 years ago. Upon physical examination, a soft solitary thyroid mass was palpated. There were no palpable neck lymph nodes. No abnormalities were found on flexible laryngoscopy. Laboratory examination showed normal levels of serum TSH, T3, and T4. The CT scan revealed a thyroid mass originating from the left lobes and extending into the mediastinum. The caudal end was situated above the superior edge of the aortic arch (shown in Fig. 1a, b). The width between the caudal ending, and the sternum notch was $37 \mathrm{~mm}$. The goiter compressed the trachea, but there was no apparent invasion of the surrounding tissues. Fine-needle aspiration cytology findings were consistent with the Papanicolaou class IIIa (shown in Fig. 2a) [4]. No follicular tissue dysplasia was noted, suggesting the diagnosis of adenomatous goiter (shown in Fig. 2b).

Although adenomatous goiter is a benign neoplasm, surgery was indicated for the patient because of the presence of upper respiratory symptoms secondary to the extension of the mass. Preoperative CT scan in the extended neck position was performed to decide whether sternotomy was necessary. Two rolled towels were inserted under the patient's scapula to extend the neck. In this position, the goiter was withdrawn cranially $18 \mathrm{~mm}$ from the sternal notch (shown in Fig. 1c, d).

Sternotomy is typically performed in cases wherein the goiter cannot be resected via a cervical incision. However, in this case, thyroidectomy was performed using the cervical approach. The goiter originated from the left thyroid lobe and extended between the trachea and the left common carotid artery. Adhesion to the surrounding tissues was not observed. The goiter was resected with the lower pole bluntly dissected (shown in Fig. 3a) to enable its mobility (shown in Fig. 3b). The recurrent laryngeal nerve and the parathyroid glands were preserved, and the goiter was successfully resected without sternotomy (shown in Fig. 3c). Pathological studies reported an adenomatous goiter not suspicious for malignancy (shown in Fig. 3d).

The patient was discharged from the hospital 8 days after the operation without complications, such as recurrent nerve paralysis and hypocalcemia. In addition, the upper respiratory symptoms were relieved. Thyroid and lung tumors were not detected during the 2-year follow-up.

\section{Discussion/Conclusion}

Substernal goiters have been variably defined, with $>50 \%$ of goiters extending into the intrathoracic region and some extending into the mediastinum $[5,6]$. The negative intrathoracic pressure pulls the goiter to descend toward the less resilient mediastinum.

\section{Karger'}




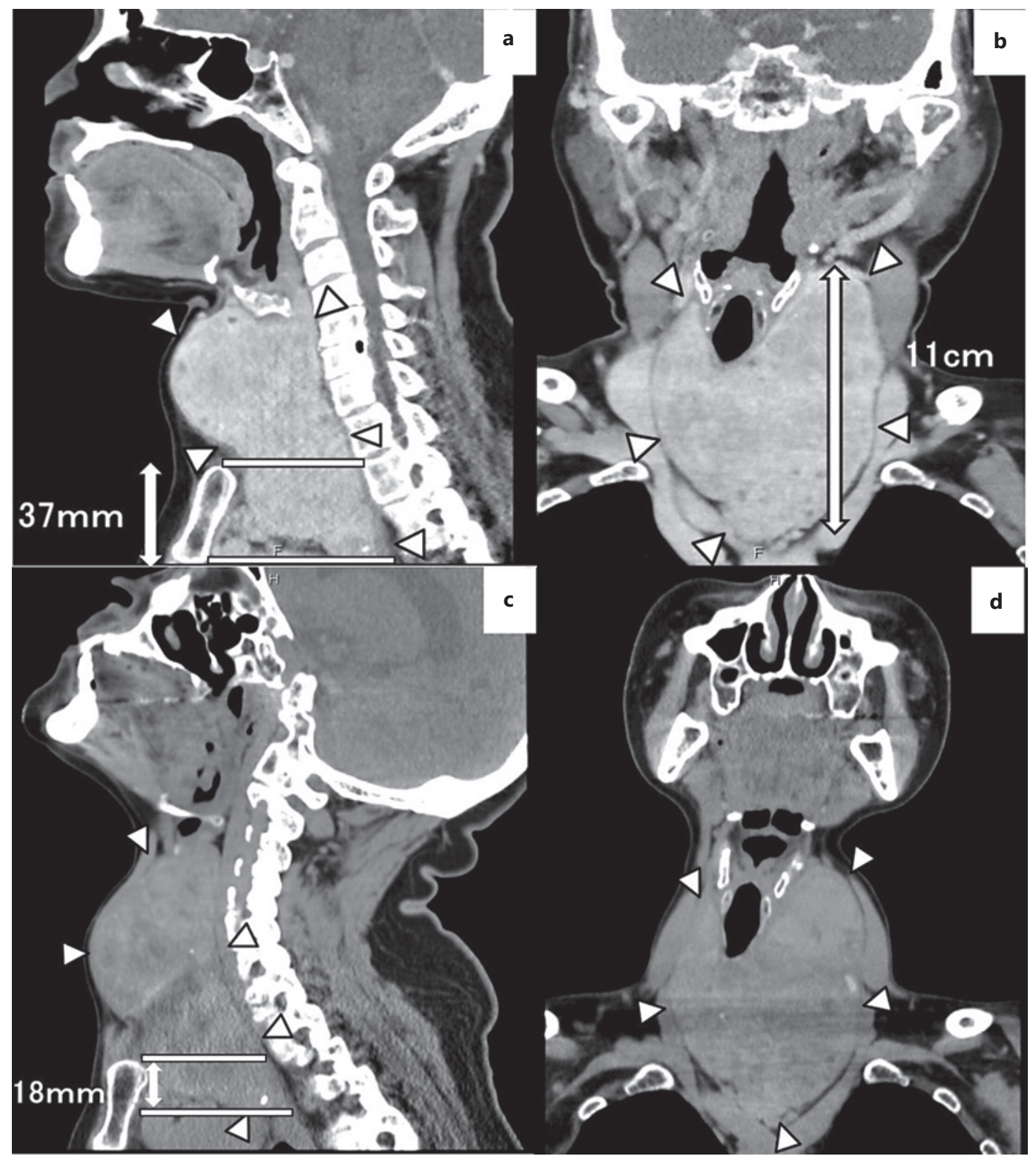

Fig. 1. Preoperative CT imaging. The arrowheads indicate the extent of the goiter. The bars show the width between the caudal endings of the goiter and sternal notch. a CT imaging (sagittal plane). The extent of the substernal goiter toward the mediastinum and the depth from the sternal notch was $37 \mathrm{~mm}$. b CT imaging (coronal plane). The 11-cm mass from the left thyroid lobe extended toward the mediastinum, and the caudal end was situated above the superior edge of the aortic arch. c CT imaging in the extended neck position (sagittal plane). The goiter was withdrawn cranially to $18 \mathrm{~mm}$ from the sternal notch. d CT imaging in the extended neck position (coronal plane). The caudal end was withdrawn above the aortic arch. CT, computed tomography.

The prevalence of substernal goiters is approximately $5.1-15.7 \%$, but its exact definition is yet to be standardized [7].

Sternotomy should be considered as an adjunct to the cervical approach in the surgical intervention of substernal goiter. Sternotomy allows proper visualization of the surgical field, which is beneficial when goiters adhere to the surrounding tissue. This method also provides safer intraoperative bleeding management. Sternotomy is indicated in cases involving possible 


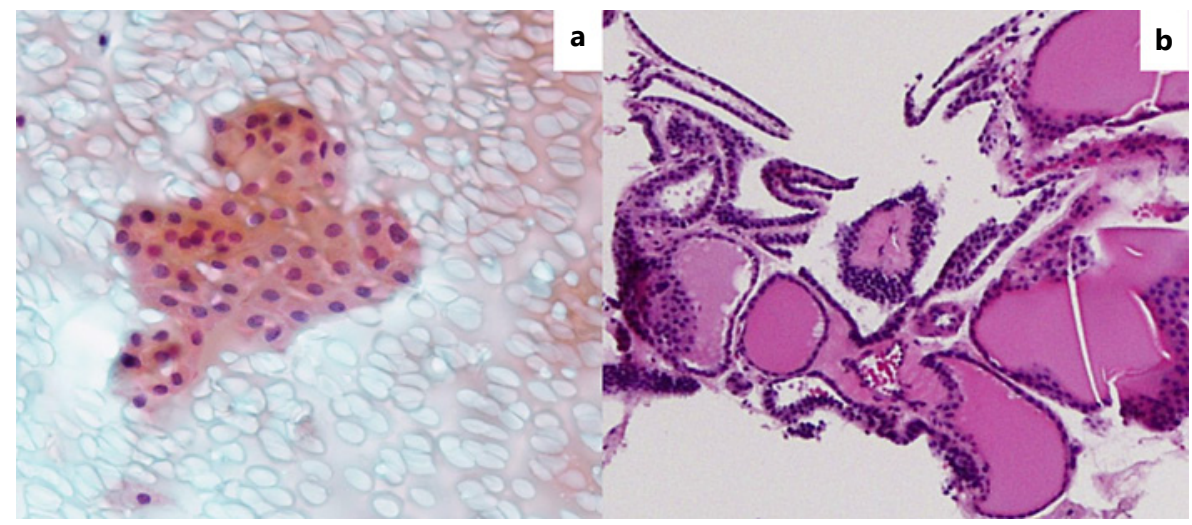

Fig. 2. Preoperative pathological examination. a Fine-needle aspiration cytology $(\times 400)$ revealed follicular cells with less dysplasia in the nuclei (Papanicolaou class IIIa). b Fine-needle biopsy $(\times 100)$ revealed a variable thyroid follicle with less dysplasia, suggesting adenomatous goiter as a diagnosis.

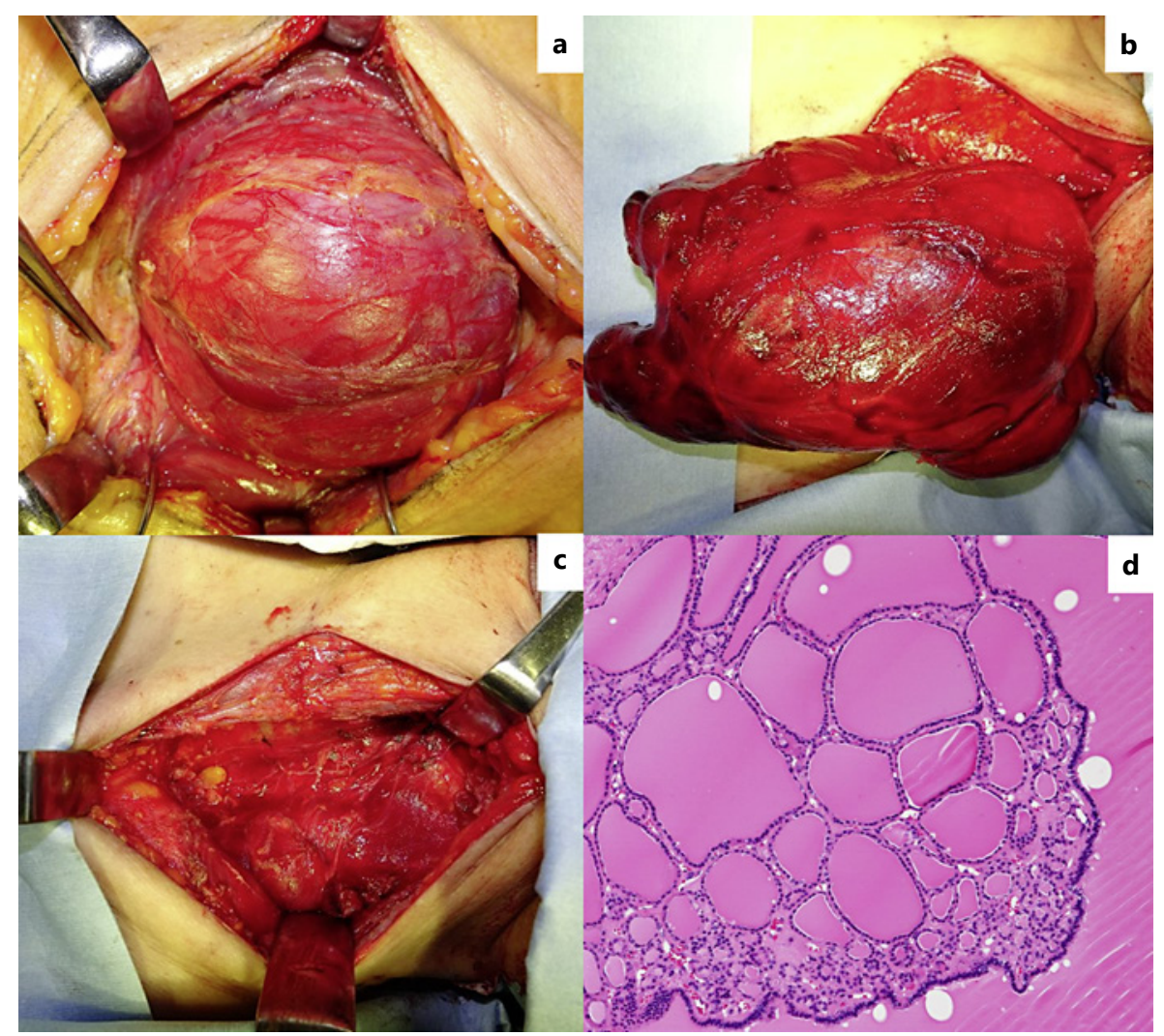

Fig. 3. Intraoperative findings and postoperative pathology. a The goiter was resected from the surrounding tissues. $\mathbf{b}$ The lower pole of the goiter was bluntly dissected and dislocated. $\mathbf{c}$ The goiter was successfully resected without sternotomy. $\mathbf{d}$ Postoperative pathology reports adenomatous goiter as a diagnosis, and no malignancy was suspected.

malignancies, relapsing goiters, large goiters that are difficult to remove with the thoracic inlet, and goiters supplied by collateral vessels [8].

A retrospective study reported that the rate of sternotomy increased as goiters reached the aortic arch, such as the case presented in this report $[9,10]$. However, there have been 
previous cases of substernal goiters reaching the aortic arch that were successfully removed without sternotomy $[11,12]$.

Goiters can be withdrawn cranially in the extended neck position during surgery, even though it appears to extend within the mediastinum. Pollard et al. [13] reported differences in the substernal extent of goiter on preoperative CT between patients with their arms overhead and those with their arms to the sides. This observation influences surgical planning.

Based on these previous studies, we conducted a preoperative CT scan in the extended neck position, which mimicked the patient's supposed position during the surgery. In the extended neck position, the goiter was located $19 \mathrm{~mm}$ superior to its position without neck extension. Therefore, surgery via the cervical approach was planned, and the goiter was successfully removed without sternotomy.

The substernal goiter was classified based on the extent of the craniocaudal plane in this report. The risk of sternotomy is increased in cases with grade 2 extension, which refers to goiters located between the convexity and concavity of the aortic arch [14]. In general, the diameter between the convexity and concavity of the aortic arch was found to be approximately $30 \mathrm{~mm}$ without any pathological expansion. The goiter was withdrawn $19 \mathrm{~mm}$ cranially in the extended neck position. The cervical approach is suitable for goiters situated approximately in the middle of the aortic arch because it is leveled down to a grade 1 extension, which is located above the convexity of the aortic arch, through neck extension. Surgery via the extracervical approach however should be considered when the cervical approach is unsuccessful. More case studies are necessary to investigate the extent of cranial goiter withdrawal in the extended neck position.

In conclusion, we reported a case of substernal goiter that was successfully removed via the cervical approach. Based on the initial imaging studies, the extracervical approach via sternotomy was necessary. However, a preoperative CT scan in the extended neck position was helpful in planning the surgical approach, which did not require sternotomy. Further research should be performed to analyze the role of preoperative CT scan in the extended neck position in determining the appropriate surgical approach.

\section{Statement of Ethics}

Study approval statement: This case study followed the protocol of the World Medical Association Declaration of Helsinki. The study is exempt from ethical committee approval since case report is exempt from committee review (Tokyo Medical University Institutional Review Board). Consent to publish statement: Informed consent, in written form, was obtained from the participants for the publication of the details of their medical case.

\section{Conflict of Interest Statement}

The authors have no conflicts of interest to declare.

\section{Funding Sources}

We received no funding for this study. 


\section{Case Reports in Oncology}

Case Rep Oncol 2021;14:1353-1358

\begin{tabular}{l|c|c|}
\hline DOI: 10.1159/000518532 & ( 2021 The Author(s). Published by S. Karger AG, Basel
\end{tabular} www.karger.com/cro

Yano et al.: Substernal Goiter Computed Tomography Findings

\section{Author Contributions}

This study was performed under the supervision of Professor Kiyoaki Tsukahara. The manuscript was drafted by Teruhisa Yano. Takuro Okada, Hiroki Sato, and Ryota Tomioka are involved in the patient treatment.

\section{Data Availability Statement}

Due to ethical restrictions, the data underlying this study are available upon reasonable request to the corresponding author.

\section{References}

1 Bürgi U, Studer H Goiter. Schweiz Med Wochenschr. 1986 Mar 15;116(11):326-31.

2 Fortuny JV, Guigard S, Karenovics W, Triponez F. Surgery of the thyroid: recent developments and perspective. Swiss Med Wkly. 2015 Jul;145:w14144.

3 Ducic Y, Oxford L. Transcervical elective superior mediastinal dissection for thyroid carcinoma. Am J Otolaryngol. 2009 Jul-Aug;30(4):221-4.

4 The Papanicolaou Society of Cytopathology Task Force on Standards of Practice. Guidelines of the papanicolaou society of cytopathology for the examination of fine-needle aspiration specimens from thyroid nodules. The papanicolaou society of cytopathology task force on standards of practice. Diagn Cytopathol. 1996 Jul; 15(1):84-9.

5 deSouza FM, Smith PE. Retrosternal goiter. J Otolaryngol. 1983 Dec;12(6):393-6.

6 Katlic MR, Grillo HC, Wang CA. Substernal goiter. Analysis of 80 patients from massachusetts general hospital. Am J Surg. 1985 Apr;149(4):283-7.

7 Nakayama H, Goda M, Kohagura K, Suganuma N, Iwasaki H, Yamazaki H, et al. A large substernal goiter that extended to both sides of the thorax. Case Rep Surg. 2018 Nov;2018:6107982. 6107982

8 Machado NO, Grant CS, Sharma AK, al Sabti HA, Kolidyan SV. Large posterior mediastinal retrosternal goiter managed by a transcervical and lateral thoracotomy approach. Gen Thorac Cardiovasc Surg. 2011 Jul;59(7): 507-11.

9 Grainger J, Saravanappa N, D’Souza A, Wilcock D, Wilson PS. The surgical approach to retrosternal goiters: the role of computerized tomography. Otolaryngol Head Neck Surg. 2015 Jun;132(6):849-51.

10 Nankee L, Chen H, Schneider DF, Sippel RS, Elfenbein DM. Substernal goiter: when is a sternotomy required? J Surg Res. 2015 Nov;199(1):121-5.

11 Rugiu MG, Piemonte M. Surgical approach to retrosternal goitre: do we still need sternotomy? Acta Otorhinolaryngol Ital. 2009 Dec;29(6):331-8.

12 Neves MC, Rosano M, Hojaij FC, Abrahão M, Cervantes O, Andreoni DM. A critical analysis of 33 patients with substernal goiter surgically treated by neck incision. Braz J Otorhinolaryngol. 2009 Mar-Apr;75(2):172-6.

13 Pollard DB, Weber CW, Hudgins PA. Preoperative imaging of thyroid goiter: how imaging technique can influence anatomic appearance and create a potential for inaccurate interpretation. AJNR Am J Neuroradiol. 2005 May;26(5):1215-7.

14 Mercante G, Gabrielli E, Pedroni C, Formisano D, Bertolini L, Nicoli F, et al. CT cross-sectional imaging classification system for substernal goiter based on risk factors for an extracervical surgical approach. Head Neck. 2011 Jun;33(6):792-9. 